\title{
Der interessante Fall
}

\section{Symptome sind Anzeichen - und bedingen eine Diagnostik!}

\author{
Wie wichtig es gerade in der \\ Geburtshilfe ist, auf Symptome \\ zu achten und wie fatal sich das \\ Ignorieren eindeutiger klinischer \\ Symptome auf den weiteren \\ Erkrankungsverlauf auswirken \\ kann, möge der folgende Fall \\ demonstrieren.
}

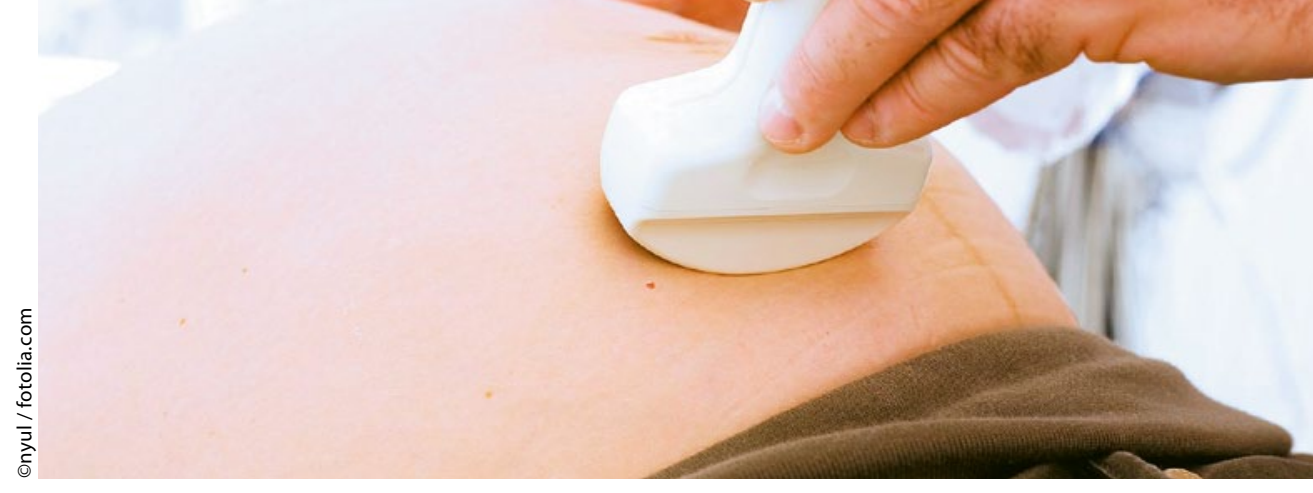

zur Einweisung von Schwangeren in Krankenhäusern der adäquaten Versorgungsstufe", die in ihrer Erstfassung bereits 1992 existierte, hätte die vorliegende Symptomatik einer Blutung und damit drohenden Frühgeburt in der 31. SSW die Verlegung in ein Pränatalzentrum Level I oder II oder eine perinatologische Schwerpunktklinik nach sich ziehen müssen.

2. Bei der Patientin bestand offenbar ein vorzeitiger Blasensprung mit anderen Infektionssymptomen, der in dem Zeitraum zwischen stationärer Aufnahme und der Entbindung aufgetreten sein muss, da bei der Aufnahmeuntersuchung die sonografisch gemessene Fruchtwassermenge nicht reduziert war und bei Entbindung ein Anhydramnion vorlag. Eine regelmäßige Wehentätigkeit muss eine regelmäßige Kontrolle der Körpertemperatur und eine laborchemische Kontrolle der Entzündungsparameter wie CRP zur Folge haben. Auf eine entsprechende Kontrolle der Entzündungsparameter und die tägliche Temperaturkontrolle wurde verzichtet. Die Persistenz der vorzeitigen Wehentätigkeit auch unter hochdosierter Tokolyse hätte als Hinweis auf ein drohendes Amnioninfektionssyndrom interpretiert werden müssen und hätte die Überwachung der La- borparameter, der Temperatur und des Ultraschalls erfordert und therapeutisch die Konsequenz der vorzeitigen Entbindung und Antibiotikagabe. Auf die eingetretende Pathologie in Form einer fetalen Tachykardie und erhöhter Körpertemperatur der Mutter um 10.00 Uhr muss unmittelbar und nicht erst mit der Entbindung drei Stunden später reagiert werden. Das Leitsymptom der fetalen Tachykardie wurde offenbar ignoriert. Nach längerer Tachykardie finden sich bei Neugeborenen nahezu viermal häufiger intrakranielle Blutungen, auch bei normalem Säure-Basis-Status. Daher sollte bei erstmaligem Auftreten der CTG-Veränderungen eine eilige, dringliche oder zügige Entbindung mit einer EE-Zeit von maximal 30 Minuten erreicht werden.

Insgesamt liegt aus gutachterlicher Sicht ein grober Behandlungsfehler vor, der aus objektiver ärztlicher Sicht nicht verständlich ist.

\section{Prof. Dr. Peter Mallmann}

Universitätsklinikum Köln Klinik und Poliklinik für Frauenheilkunde und Geburtshilfe

Kerpener Str. 34

50931 Köln der Deutschen Gesellschaft für Gynä-

kologie und Geburtshilfe „Indikation 